\title{
Sudden Unexplained Cardiac Arrest in Apparently Healthy Soldier: is Pre-Workout Supplement Safe!
}

\author{
Ibrahim Osman, MD, Bankim Patel, MD, Assad Movahed, MD \\ East Carolina University, The Brody School of Medicine, East Carolina Heart Institute \\ Department of Cardiovascular Sciences, Greenville, NC, USA \\ MOVAHEDA@ecu.edu
}

\begin{abstract}
Dietary pre workout supplements are commonly used in the US active duty population often without physician knowledge or appropriate screening. Selected dietary supplements have been associated with a number of unexplained cardiac arrest and death in service members. Many of these supplements are marketed as weight loss and athletic performance enhancement products. The association of various Ephedra-containing products with adverse cardiovascular events has led to a ban on the sale of these products by the U.S. Food and Drug Administration (FDA) in which it resulted in the emergence of a newer formulations marketed as "Ephedra-free" but contain other sympathomimetic substances, however, the safety of which has not been established. We report a case of active duty soldier who was taking commercially available dietary supplements called Cellucor C4; collapsed during routine morning exercise at his Navy base from cardiac arrest and ultimately survived. Our case highlights concerns that C4 in combination with other ingredients may be associated with significant consequences, reminiscent of previous adverse events from other sympathomimetic drugs previously removed from the market. Health care professionals, should be aware of the potential risk of these supplements and be prepared to discuss these risks with their patients.
\end{abstract}

\section{INTRODUCTION}

The use of pre-workout supplement (PWS) is common in the active duty population, with $50 \%$ to $87 \%$ of soldiers reporting regular use.1-3 These supplements often contain long lists of vitamins, amino acids, energy supplements, and herbal formulations in their ingredient list. However, under the Dietary Supplement and Health Education Act of 1994, these products are not considered drugs, and therefore are not required to demonstrate purity, efficacy, or safety before distribution. These products are available without a prescription and without physician knowledge or approval. As a result, the individuals taking PWS may not be aware of the significant health risks associated with them. Furthermore, although physicians are aware of many of the signs and symptoms of adverse events due to PWS, very few of these events are reported.1 This impairs public health efforts to identify and monitor potentially dangerous products currently on the market. Previous case reports of adverse events associated with PWS use in the active duty population have included acute liver damage, 4,5 rhabdomyolysis, 6 ischemic colitis, 7 acute psychosis, 8 and ventricular fibrillation.

\section{Case Presentation}

A 23-year-old male navy soldier who was in excellent physical condition (last physical fitness test placed him above the 90th percentile), suffered a sudden cardiac arrest while running in formation with his battalion for about 5 minutes, was found to be pulseless; bystanders initiated CPR with the return of spontaneous circulation within 5 minutes, he subsequently developed 3 episodes of ventricular fibrillation (V-FIB) on route to the emergency department which was successfully defibrillated and aggressive resuscitation efforts were applied including active core cooling via an endovascular device, endotracheal intubation, and fluid restoration. He smokes half pack per day, occasional marijuana use and social drinking. He has a paternal grandmother who 
had a heart attack at her 30 . He described the daily exercise regimen composed of weight training and aerobic activity approximately five times a week of varying time length; prior to each session, he ingested a caffeine-containing drink (Red Bull) and pre workout supplement called Cellucor C4. On the day of the cardiac arrest, he mixed the Red Bull and approximately one serving of Cellucor C4 with the engagement of his exercise routine. Upon arrival at the hospital, physical examination was remarkable for hyperthermia per protocol, intubated, blood pressure $127 / 70 \mathrm{mmHg}$, pulse 66 , respiratory rate 17 , and saturating at $97 \%$. Electrocardiogram (ECG) showed normal sinus rhythm, right axis deviation and small positive deflection ('blip') buried in the end of the QRS complex in lead V1, II, III and aVF suggestive of epsilon wave. Initial laboratory values were remarkable for a troponin I of $0.04 \mathrm{ng} / \mathrm{mL}$, white blood cell count of 18000. Toxicology analyses were negative. Laboratory evaluation revealed no findings consistent with a hypercoagulable state, nor was there other evidence of systemic thromboembolic disease.

Transthoracic echocardiogram showed a normal left ventricle function. Coronary angiography was performed showing normal coronary arteries. A cardiac MRI showed no evidence of myocardial fibrosis or Arrhythmogenic right ventricular cardiomyopathy (ARVC). Subsequent cardiac genetic testing was done without yield. He was eventually extubated and transferred to our step down unit. Cardiac electrophysiology (EP) study was performed without inducible arrhythmias and ultimately underwent an ICD placement for secondary prevention.

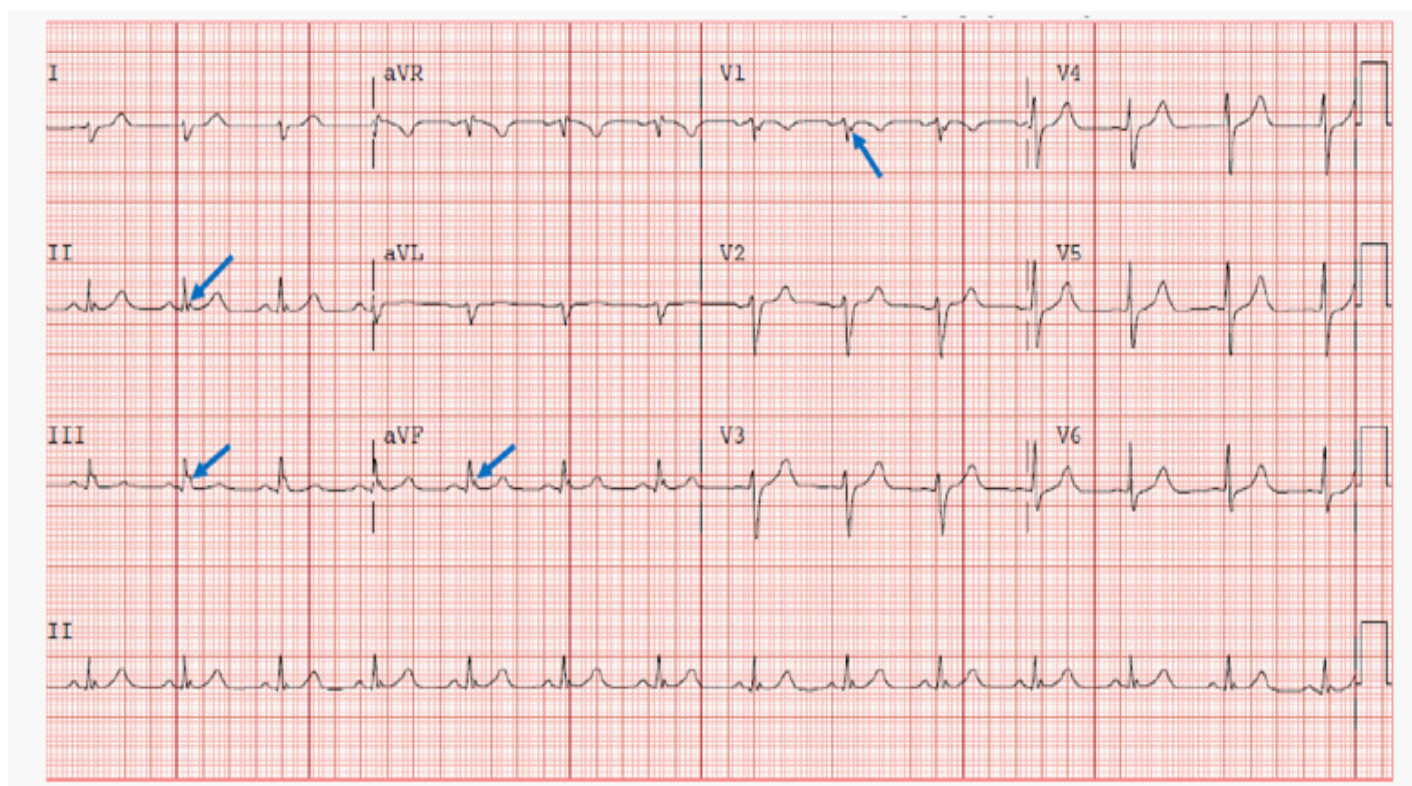

Fig1. Electrocaralogram ( $E(G)$ showea normal sinus rnythm, right axis aeviation ana small positive aeflection ('blip') buried in the end of the QRS complex in lead V1, II, III and aVF suggestive of epsilon wave (Arrow)

\section{Discussion}

Nutrient timing refers to the methodical, timed ingestion of carbohydrate, protein, fat and other dietary supplements either before, during, or after physical activity [10]. Supplementation during the period immediately preceding physical activity has become an increasingly popular strategy among competitive and recreational athletes alike as a means of improving performance [11]. In response to this trend, manufacturers have developed pre-workout supplements (PWS), which typically combine caffeine with any number of purported ergogenic substances, such as dimethylamylamine, creatine, arginine, $\beta$-alanine, taurine, nitrate and phosphates. As the number of PWS (unregulated dietary supplements) available on the market grows, each containing their own "proprietary blend" of active ingredients, it must be determined which, if any, are safe for chronic consumption. 
This becomes particularly important as concerns have arisen over the concept of proprietary blends, namely the fact that the FDA does not monitor the amounts of ingredients used in these blends or the accuracy of product labeling by manufacturers [11]. While some dietary supplement labels instruct consumers to seek the advice of a health care professional before using the products, the labels usually do not disclose all ingredients or their precise amounts, and evidence to support the purported performance-enhancing benefits is generally lacking. There is limited evidence to support the use of some pre-workout supplement ingredients. For example, in one small placebo-controlled study ( $n=12$ ), the use of the energy drink Red Bull (containing caffeine and taurine) 40 minutes before a simulated cycling time trial appeared to provide a meaningful ergogenic benefit; in another small study $(n=12)$, the use of a similar caffeine-containing product (Redline) by strength-trained athletes was found to improve reaction time, energy, and mental focus relative to placebo use. However, published evidence on the use of the other ingredients listed above is scant, inconclusive, or conflicting. Adverse effects reported in association with pre-workout supplements include gastrointestinal symptoms, cardiac arrhythmia, blood pressure increases, and potential effects on lipids and blood glucose.

Although an expanding body of literature reports adverse cardiovascular outcomes in association with the use of synephrine-containing products, our case report is, to our knowledge, the first in which active duty soldier with no history of identifiable cardiovascular risk factors experienced V-FIB arrest shortly after ingesting a Cellocur C4. While the cause of his SCA remains uncertain, it is plausible that the pre-workout C4 might triggered his V-FIB event as it contains a variety of sympathomimetic and stimulant compounds.

The reported specificity qualified the epsilon wave not only as a major criterion for ARVC diagnosis but also as a hallmark of the disease. It is, however, a relatively rare finding, manifesting itself only in from $1 \%$ to $25 \%$ of ARVC patients [12]. It is important to acknowledge that the epsilon wave is not specific to ARVC only and has been reported in patients with other conditions such as sarcoidosis [13], myocarditis [14], or acute RV infarction [15]. Moreover, an iatrogenic form of epsilon wave has been described, occurring after catheter ablation in the RV [16].

Our case highlights the potential health hazards associated with athletic-performance-enhancing and weight-reduction supplements that contain sympathomimetic compounds similar to ephedra. In light of these facts, as well as the empiric risk of synergism in the above-mentioned agents, we believe that performance enhancement use was the precipitant of this patient's cardiac event, and we believe that greater FDA and some government agencies such as US military involvement in the regulation of such supplements is warranted.

\section{CONC LUSION}

Although evidence exists to support the performance-enhancement efficacy of some pre-workout ingredients as standalone agents, published data on combination products are scant, inconclusive, or conflicting. The safety of these products may be compromised if users consume larger-than-recommended amounts or use more than one product. Thus, the safety of Cellocur $\mathrm{C} 4$ remains an open question and needs more investigation. Regulatory action may be necessary.

\section{REFERENCES}

1. Cellini M, Attipoe S, Seales P, et al: Dietary supplements: physician knowledge and adverse event reporting. Med Sci Sports Exerc 2013; 45(1): 23-8.

2. Lieberman HR, Stavinoha TB, McGraw SM, White A, Hadden LS, Marriott BP: Use of dietary supplements among active-duty US Army soldiers. Am J Clin Nutr 2010; 92(4): 985-95.

3. Bovill ME, Tharion WJ, Lieberman HR: Nutrition knowledge and supplement use among elite U.S. Army soldiers. Mil Med 2003; 168(12): 997-1000.

4. Hughes J, Shelton B, Hughes T: Suspected dietary supplement injuries in special operations soldiers. J Spec Oper Med 2010; 10(3): 14-24. 
Sudden Unexplained Cardiac Arrest In Apparently Healthy Soldier: Is Pre-Workout Supplement Safe!

5. Jones FJ, Andrews AH: Acute liver injury associated with the herbal supplement Hydroxycut in a soldier deployed to Iraq. Am J Gastroenterol 2007; 102(10): 2357-8.

6. Burke J, Seda G, Allen D, Knee TS: A case of severe exercise-induced rhabdomyolysis associated with a weight-loss dietary supplement. Mil Med 2007; 172(6): 656-8.

7. Magee CD, Moawad FJ, Moses F: NO-Xplode: a case of supplementassociated ischemic colitis. Mil Med 2010; 175(3): 202-5.

8. Boos CJ, White SH, Bland SA, McAllister PD: Dietary supplements and military operations: caution is advised. JR Army Med Corps 2010; 156(1): 41-3.

9. Stephensen TA, Sarlay R Jr: Ventricular fibrillation associated with use of synephrine containing dietary supplement.MilMed 2009; 174(12): 1313-9.

10. Kerksick C, Harvey T, Stout J, Campbell B, Wilborn C, Kreider R, et al. International society of sports nutrition position stand: nutrient timing. J Int Soc Sports Nutr. 2008;5:17. doi: 10.1186/1550-2783-5-17. [PMC free article][PubMed] [Cross Ref]

11. Eudy AE, Gordon LL, Hockaday BC, Lee DA, Lee V, Luu D, et al. Efficacy and safety of ingredients found in preworkout supplements. Am J Health Syst Pharm. 2013;70:577-88. doi: 10.2146/ajhp120118.[PubMed] [Cross Ref]

12. Efficacy and safety of ingredients found in preworkout supplements. Anne E. Eudy, Lindsay L. Gordon, Brandon C. Hockaday, Daniel A. Lee, Vivianne Lee, Daniel Luu, Carlos A. Martinez and Peter J. Ambrose American Journal of Health-System Pharmacy April 2013, 70 (7) 577-588; DOI: https://doi.org/10.2146/ajhp120118

13. Platonov PG, Calkins H, Hauer RN, et al. High interobserver variability in the assessment of epsilon waves: Implications for diagnosis of arrhythmogenic right ventricular cardiomyopathy/dysplasia. Heart Rhythm. 2016; 13(1): 208-216, doi: 10.1016/j. hrthm.2015.08.031, indexed in Pubmed: 26304715.

14. Nery PB, Keren A, Healey J, et al. Isolated cardiac sarcoidosis: establishing the diagnosis with electroanatomic mapping-guided endomyocardial biopsy. Can J Cardiol. 2013; 29(8): 1015. e1-1015.e3, doi: 10.1016/j. cjca.2012.09.009, indexed in Pubmed: 23246240.

15. Vollmann D, Goette A, Kandolf R, et al. Epsilon waves in giant-cell myocarditis. Eur Heart J. 2014; 35(1): 9, doi: 10.1093/eurheartj/eht338, indexed in Pubmed: 23990602.

16. Zorio E, Arnau MA, Rueda J, et al. The presence of epsilon waves in a patient with acute right ventricular infarction. Pacing Clin Electrophysiol. 2005; 28(3): 245-247, doi: 10.1111/j.1540-8159. 2005.40021.x, indexed in Pubmed: 15733188.

17. Caldwell J, Redfearn D, Chiale PA, et al. Ablation-induced epsilon wave. Heart Rhythm. 2013; 10(11): 1737-1738, doi: 10.1016/j. hrthm.2012.08.038, indexed in Pubmed: 23102627.

Citation: Ibrahim Osman, MD, Bankim Patel, MD, Assad Movahed, MD, “Sudden Unexplained Cardiac Arrest in Apparently Healthy Soldier: is Pre-Workout Supplement Safe!". American Research Journal of Cardiovascular Diseases, 1(1); pp:17-20

Copyright @ Ibrahim Osman, MD, Bankim Patel, MD, Assad Movahed, MD, This is an open access article distributed under the Creative Commons Attribution License, which permits unrestricted use, distribution, and reproduction in any medium, provided the original work is properly cited.

American Research Journal of Cardiovascular Diseases

Page 20 